\title{
A liquid progressive multifocal lens adjusted by the deformation of a non-uniform elastic membrane due to the variation of liquid pressure
}

Weiliang Jia, Dong Xiang ${ }^{*}$ and Songjing Li ${ }^{*}$

\begin{abstract}
Background: In this paper, a liquid progressive multifocal lens with solid-liquid structure is demonstrated, which mainly consists of two elastic polydimethylsiloxane (PDMS) membranes, a solid substrate and liquid.

Methods: To realize the adjustment of the focuses progressively, the thickness of one of the membrane is designed non-uniform. By controlling the liquid pressure working on the membranes, the curvature of the membrane can be changed continuously and the power of the lens can be altered simultaneously. In this paper, the structure and a fabrication method of the lens is introduced, and a power distribution model is built for the calculation of the power distribution characteristics. Moreover, the deformation of the non-uniform elastic membrane of the lens under different pressures is analysed with finite element method (FEM).

Results: Finally, a prototype of the lens is developed and tested by applying a micro laser displacement sensor, and it is demonstrated that the progressive multifocal lens is feasible.

Conclusion: A novel liquid progressive multifocal lens with a non-uniform thickness elastic membrane is proposed. From the simulation and experimental investigation, it can be concluded that the proposed liquid lens can realize progressive multifocal through using non-uniform elastic membrane and the power can be adjusted by the pressure which is controlled by the liquid volume filled in the lens.
\end{abstract}

Keywords: Liquid lens, Progressive multifocal lens, Non-uniform PDMS membrane, Power distribution

\section{Background}

Progressive multifocal lens is commonly used to compensate presbyopia caused by losing ocular accommodation, especially happened on people over the age of 40 who can hardly see nearby objects clearly. As progressive multifocal lens provides a whole clear visual field from distant to near ranges comparing with the traditional bifocal and multifocal lens, it attracts more and more attentions in academic and commercial fields.

Nowadays, although there are already several kinds of solid progressive multifocal lens or progressive addition lens in the market, the method to realize progressive multifocal adjustment mechanism is still under research.

\footnotetext{
* Correspondence: xiangdong@hit.edu.cn; lisongjing@hit.edu.cn Department of Fluid Control and Automation, Harbin Institute of Technology, Harbin 150001, China
}

Progressive multifocal lens can distributes the power of the three important zones namely far view zone, near view zone and intermediate zone progressively and smoothly [1]. G. Savio, G. Concheri and R. Meneghello designed a progressive lens applying discrete shape modelling techniques which considered both optical and geometrical parameters [2]. Likewise, Wei-Yao Hsu, Yen-Liang Liu and Yuan-Chieh Cheng proposed a progressive multifocal lens surface with a B-spline description optimizing from the average power of the far and near view zones [3]. Then a variational-difference numerical method for designing the optical freeform surface of a progressive multifocal lens was introduced, it can minimize the functional directly by finite difference method rather than approximate the solution of the corresponding Euler-Lagrange equation to the functional 
[4]. Moreover, a multioptical-axis mathematical model based on the human eye in the different observation orientations was developed to design the freeform progressive multifocal lens through theoretical and experimental investigations [5].

However, as the visual requirements of spectacle lens wearers differ greatly, the traditional "one-size-fits-all" progressive lens design framework is no longer suitable for every progressive lens wearer. Therefore, designers need to tailor more suitable spectacle lenses for the unique visual requirements. Liquid adjustable lens have been studied in recent years due to the advantages that the focus adjustment can easily be realized by the volume variation of liquid to meet the need of the wears at any circumstances.

Several studies on liquid-filled lens have been reported. An asymmetrical liquid-filled lens was designed, of which both the light direction and the focal length can be changed by controlling the tilt angel of the pressure ring and the hydraulic pressure of the lens [6]. Likewise, a variable focus membrane lens was presented which focused on the problem of theoretical evaluation of the surface shape using the generalized nonlinear differential equation [7]. In addition, a liquid-filled tunable focus microlens was designed by using a membrane with different thickness between the central and peripheral region [8]. Besides, many kinds of liquid-filled lens with different actuators or control strategies were also designed in recent years, furthermore, the liquid tunable aspherical lenses are formed by liquid sealed with a non-uniform thickness membrane which could alleviate the edge clamping effect and reduce spherical aberration [9-15]. In order to decrease the influence of gravity to the performance of liquid lens, one of a method is using an in-plane pretention force to a membrane to take the place of surface tension and the other is using a non-uniform thickness profile of the flexible membrane $[16,17]$. However, one of the common disadvantages of these liquid-filled lenses is that the focus could be adjusted in one region or only have one focus. Therefore, to suit different groups of people under different conditions, a liquid progressive multifocal lens with a non-uniform thickness elastic membrane is designed in this paper.

The aim of this paper is to study the structure of liquid progressive multifocal lens, the deformation characteristics of non-uniform elastic membrane and the power distribution characteristics of the lens. The power distribution model is established for the calculation of the power distribution characteristics and the surface deformation of the lens is analyzed under different pressures with finite element method. Besides, a prototype is developed and tested by using a micro laser displacement sensor to demonstrate the feasibility of the liquid progressive multifocal lens.

\section{Methods}

\section{Structure and fabrication}

In this section, the liquid progressive multifocal lens is introduced in detail, which consists of two elastic membranes and a solid substrate as shown in in the Fig. 1a of Fig. 1b. The thickness of one membrane is designed non- uniform and the other is constant. They are clamped together at the edge to form a closed cavity defining the lens structure with an aperture size of $30 \mathrm{~mm}$. In this paper, the power of the lens is designed to distribute between +4 and +6 (focal lengths are $1 / 4 \mathrm{~m}$ and $1 / 6 \mathrm{~m}$ ) from the top region to the bottom. The cavity constrained on the substrate is connected with an external pumping system via a pinhole, through which the liquid can flow in or out of the chamber. As the elastic membrane with non-uniform thickness will deform differently at top and bottom regions under the same pressure of liquid inside the cavity, the corresponding surface curvature and the focal lengths of the lens would change.

In the structure design of the lens, the solid substrate is made of PMMA, which has a refractive index of 1.49 , a haze of $0.5 \%$ and light transmission of $92 \%$. The two elastic membranes are made of PDMS, a mixture of a Sylgard 184 silicone elastomer and a base curing agent from the Dow Corning Corporation (Midland, Michigan) in a 10:1 weight ratio. After being cured at $75{ }^{\circ} \mathrm{C}$ for $40 \mathrm{~min}$, the PDMS become a solid with the density of $0.975 \mathrm{~g} / \mathrm{cm}^{3}$, Young's modulus of $1.2 \mathrm{MPa}$, Poisson ratio of 0.49 , and the refractive index of 1.40 .

In addition, an immersion oil is applied as the liquid of the lens with high refractive index $(n=1.51)$, large range of dynamic viscosity and it has the ability to reduce the evaporation. For a liquid with a higher index, a larger focal length can be obtained from a smaller volume displacement due to the deformation of elastic membrane, thus, the lens aberration will decrease [18]. By tuning the volume of the liquid in the cavity of the lens via the pinhole, one can continuously change the curvature of the elastic membrane to form the shape of the lens.

The fabrication of the liquid lens can be realized by the formation of elastic membranes and the bonding between the membranes and the solid substrate. A mould is designed to make the non-uniform thickness elastic membrane for the lens and a process including mixing, stirring, degassing and heating for curing is required. After the surface treatment by the oxygen plasma, the two treated elastic membranes are placed and their edges are kept together tightly for about $24 \mathrm{~h}$. Then a reliable connection without leakage can be achieved. By using an adhesive film (ARclear ${ }^{\circ}$ Optically clear adhesive 8154, Adhesive Research, Glen Rock, PA USA), the 

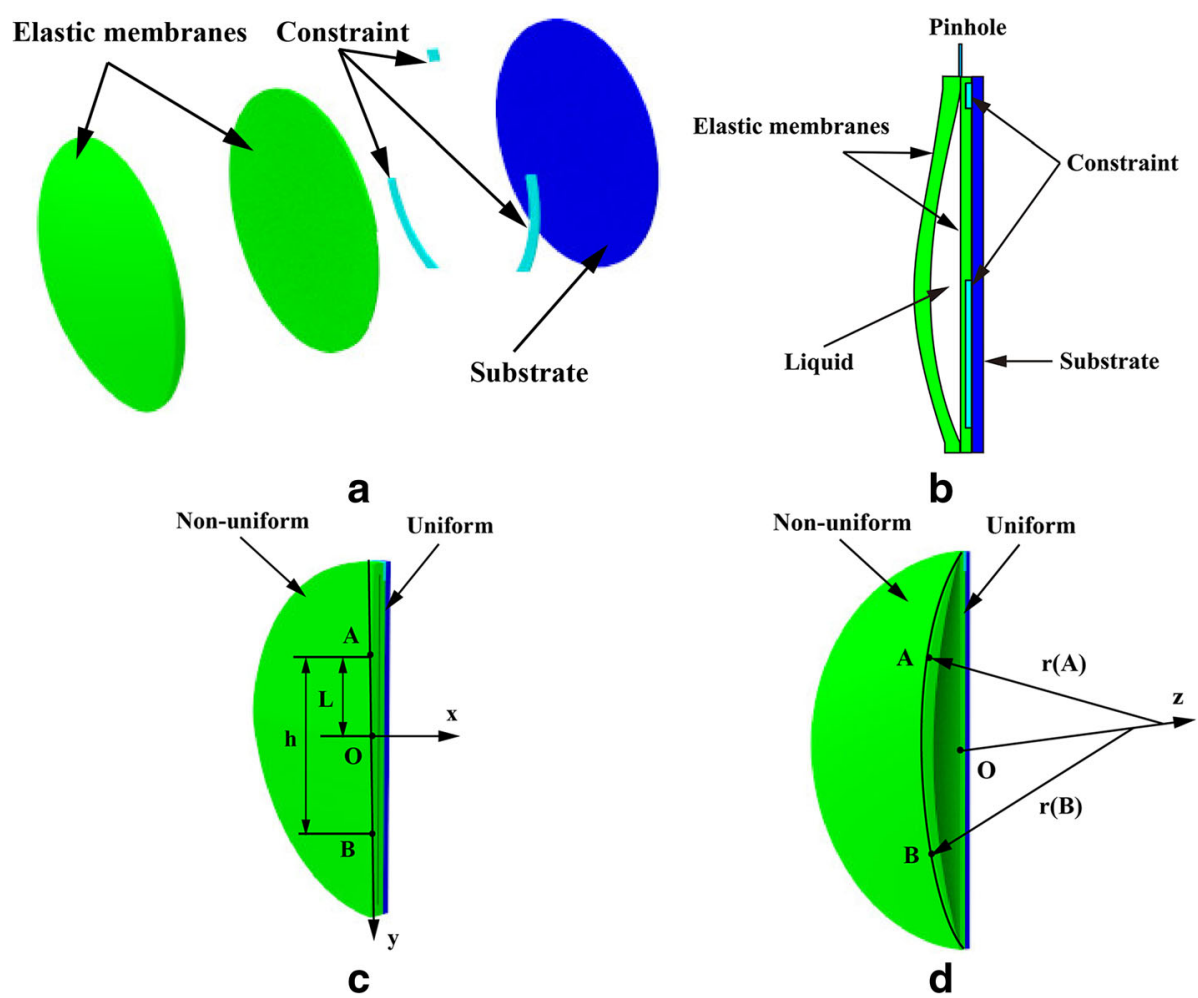

Fig. 1 Scheme of a liquid-filled progressive multifocal lens: a schematic exploded diagram of the lens, $\mathbf{b}$ the cross-sectional view of schematic structure, $\mathbf{c}$ the cross-sectional view before injecting liquid and (d) the cross-sectional view after injecting liquid

uniform thickness membrane and the substrate can also bond together tightly $[19,20]$.

\section{The power distribution model and characteristics}

The liquid progressive multifocal lens is designed based on the definition of the ideal optical profile. The elastic membrane's deformation of an aspherical lens can be expressed by the following equation [21]:

$$
\begin{aligned}
& \frac{1}{r(u)}=\frac{1}{r_{\mathrm{A}}}+\left(\frac{1}{r_{B}}-\frac{1}{r_{A}}\right) \sum_{n=m}^{m+l-1} c_{\mathrm{n}}(u+L)^{n} \\
& \left\{\begin{array}{l}
r(u)_{u=-L}=r_{A} \\
r(u)_{u=h-L}=r_{B}
\end{array}\right.
\end{aligned}
$$

where, $u$ is the coordinate at the meridian line, $r(u)$ is the curvature radius of the progressive surface at the coordinate $u, r_{A}$ and $r_{B}$ are the curvature radius of the progressive surface at the distance point $A$ with a relative lower mean power and the near point $B$ with a relative higher mean power respectively shown in the Eq. (2), $h$ is the vertical displacement between point $A$ and point $B, L$ represents the vertical displacement of distance point above central point, as shown in the Fig. 1c of Fig. 1d. $m$ is the lowest order of Eq. (1) when $\frac{d^{n}}{d u^{n}}\left(\frac{1}{r(u)}\right)$ exists the first non-zero at the distance point $A . l$ is the order when $\frac{d^{n}}{d u^{n}}\left(\frac{1}{r(u)}\right)$ exists the first non-zero at the near point $B$ and the coefficients $c_{n}$ can be got by solving Eq. (3).

The criterion of designing the meridian power law is as following, firstly, the variation of the curvature from the distance point to the near point should be slow. Secondly, the radius of curvature along the meridian changes progressively. The goal of designing meridian power law is to solve the Eq. (3) [22]. $n=1,2, \ldots \ldots, l-1$.

$$
\begin{gathered}
\left.\frac{d^{n}}{d u^{n}}\left(\frac{1}{r(u)}\right)\right|_{u=h-L}=0 \\
\sum_{n=m}^{m+l-1} c_{n} h^{n}=1
\end{gathered}
$$

In this paper, the power of the progressive surface at the distance point $A$ is set to $D_{A}=+4$, the power of the progressive surface at the near point $B$ is set to $D_{B}=+6$, the vertical displacement $L$ and $h$ are set to $L=9 \mathrm{~mm}$ and $h=20 \mathrm{~mm}$, respectively. As the coordinate $u$ along the contour is equal to the value at the point of intersecting with the meridian, the value of $u$ at an arbitrary coordinate $(x, y)$ can be calculated with the values $x$ and $y$. Thus, the lens surface height $z(x, y)$ can be specified by the following equation [23]. 


$$
\mathrm{z}(x, y)=\zeta(u)-\left\{r(u)^{2}-[x-\xi(u)]^{2}-y^{2}\right\}^{1 / 2}
$$

where,

$$
\begin{aligned}
& \xi(u)=u-r(u) \sin \theta(u) \\
& \zeta(u)=r(u) \cos \theta(u)+\int_{0}^{u} \tan \theta(u) d u \\
& \sin \theta(u)=\int_{0}^{u} \frac{d u}{r(u)}
\end{aligned}
$$

As the liquid filled in the lens immersion oil whose refractive index is known, through calculating the mean curvature solving rules according to Eqs. 5, 6 and 7, the power distribution of the progressive multifocal lens can be got [24].

$$
D=1000(n-1) \frac{k_{1}+k_{2}}{2}=1000(n-1) H
$$

where $k_{1}$ and $k_{2}$ are the orthogonal curvature for any point on the surface of lens, which satisfy the quadratic equations as following:

$$
\begin{aligned}
& g^{4} k^{2}+g\left[2 p q s-\left(1+p^{2}\right) t-\left(1+q^{2}\right) r\right] k+\left(r t-s^{2}\right) \\
& \quad=0 \\
& H=\frac{k_{1}+k_{2}}{2}=\frac{\left(1+p^{2}\right) t+\left(1+q^{2}\right) r-2 p q s}{2 g^{3}}
\end{aligned}
$$

where $H$ denotes the mean curvature of the point, and

$$
\begin{gathered}
p=\frac{\partial z}{\partial x}, \quad q=\frac{\partial z}{\partial y}, \quad r=\frac{\partial^{2} z}{\partial x^{2}}, \quad s=\frac{\partial^{2} z}{\partial x \partial y}, \quad t=\frac{\partial^{2} z}{\partial y^{2}}, \quad g= \\
\sqrt{1+p^{2}+q^{2}}
\end{gathered}
$$

Through calculating, the power distribution of the progressive multifocal lens is shown in Fig. 2. It can be seen that the power of the progressive surface at the distance point $A$ is $D_{A}=+3.96$ and the power of the progressive surface at the near point $B$ is $D_{B}=+6.02$ in this paper. In addition, the power distribution of the lens changes progressively from the distance point $A$ to the near point $B$.

\section{The deformation characteristics of a non-uniform elastic membrane due to different liquid pressure}

In order to analyze the deformation characteristics of a non-uniform elastic membrane, the finite element method (FEM) based on ANSYS Multiphysics software is applied in this paper. For the design of the lens, PDMS is used as the material of the membrane, therefore, the Young's modulus, Poisson's ratio, density and the refractive index of the elastic membrane in the analysis are set to be $1.2 \mathrm{MPa}, 0.49,0.982 \mathrm{~g} / \mathrm{cm}^{3}$ and 1.40 , respectively. The most important factors considered in the deformation analysis of the elastic membrane are the

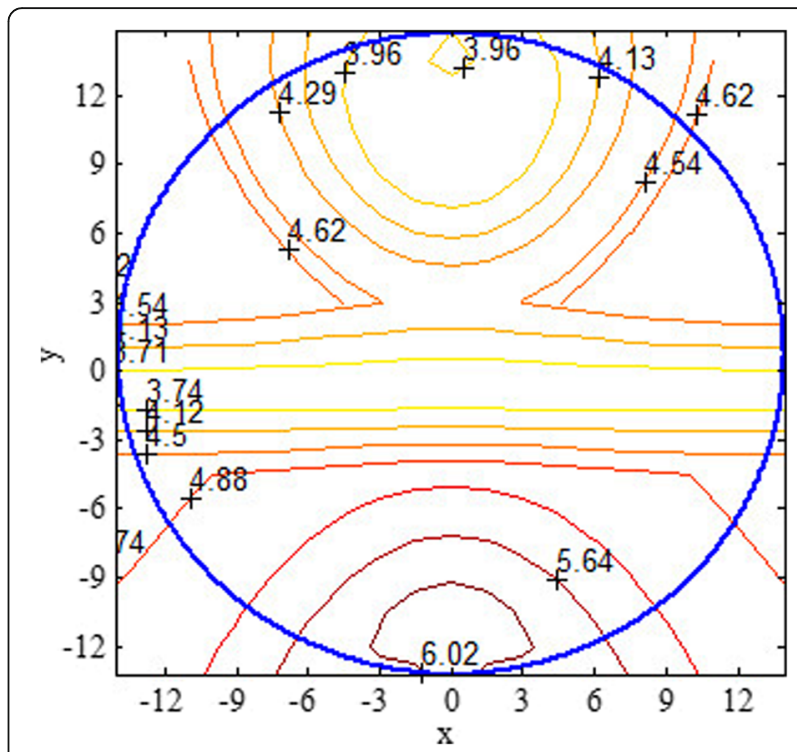

Fig. 2 The power distribution of the progressive multifocal lens

thickness distribution of the elastic membrane, liquid pressure and the boundary constraint on the membrane,

The analysis process included building a non-uniform thickness membrane model by Solidworks ${ }^{\circledR}$ software, transferring the model file to ANSYS for meshing and analysis, and recording the data for the deformed profiles. Then the raw data are analyzed by Microsoft Excel and Matlab to reveal the membrane deformation profile and the mean power distribution of the surface.

The geometric model and the constraint of the non-uniform thickness elastic membrane is illustrated in Fig. 3 and the unit of presented numbers in the Figure is millimeter $(\mathrm{mm})$. The thickness at the center region is designed to be larger than the other regions, moreover, the changing rates of the thickness on the top and the bottom regions are different. The diameter of the non-uniform thickness membrane is set to $30 \mathrm{~mm}$ and the thickness is set to $1 \mathrm{~mm}$ at the center region, furthermore, the edge thicknesses of the top and bottom are set to $0.56 \mathrm{~mm}$ and $0.71 \mathrm{~mm}$ respectively, the changing trend of the thickness of the membrane among the three regions is gradient. The constraint are three parts of edge clamped which located at the top and two sides of the membrane.

In the deformation analysis of the non-uniform thickness membrane, the three-dimensional geometric model of the membrane is uploaded into the ANSYS software together with the material properties and boundary constraint, then the membrane meshing is done for the static analysis under different pressures. The simulation results of the membrane deformation on the pressures of $104 \mathrm{~Pa}, 90 \mathrm{~Pa}, 100 \mathrm{~Pa}$ and $110 \mathrm{~Pa}$ are presented in Fig. 4, which mainly contain the maps in the view of horizontal and longitudinal. In the simulation, DMX is 


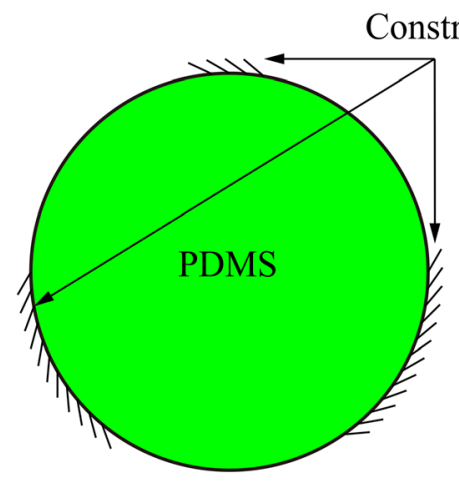

a
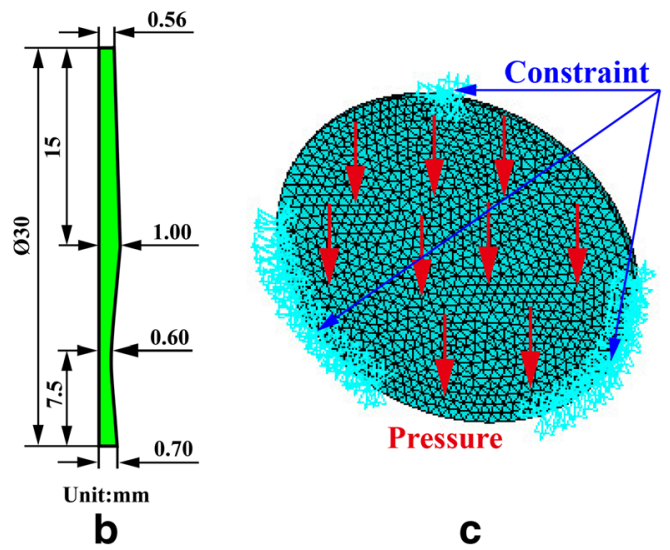

Fig. 3 Geometry of the non-uniform membrane (a) longitudinal model with constraint, b Horizontal model, c Mesh model with pressure and constraint

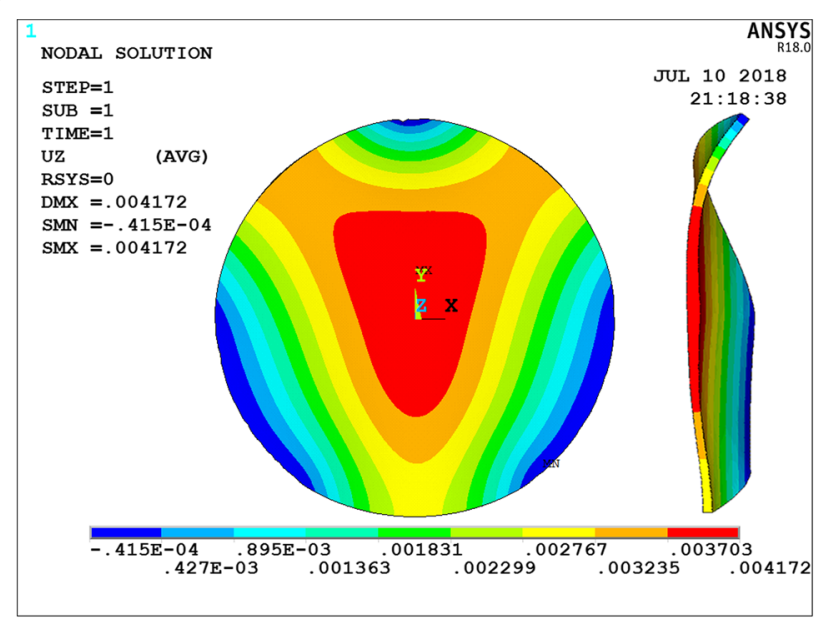

a

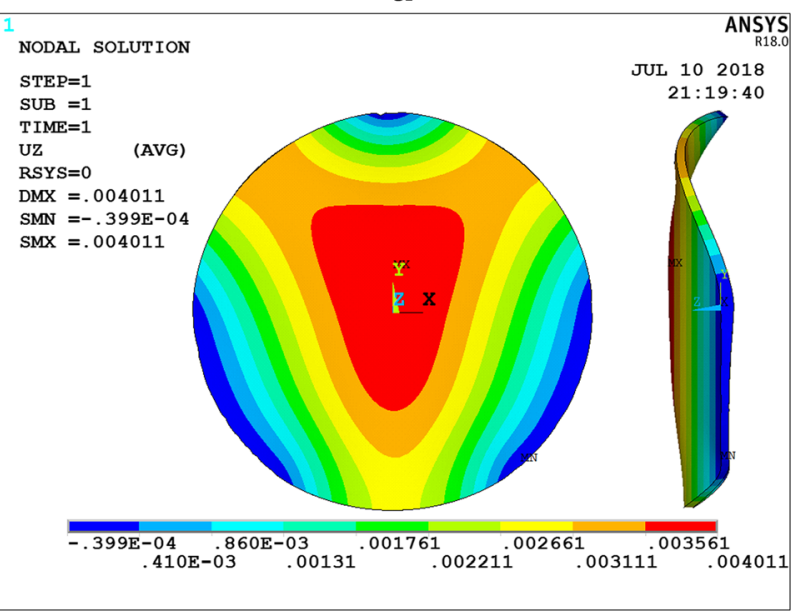

C

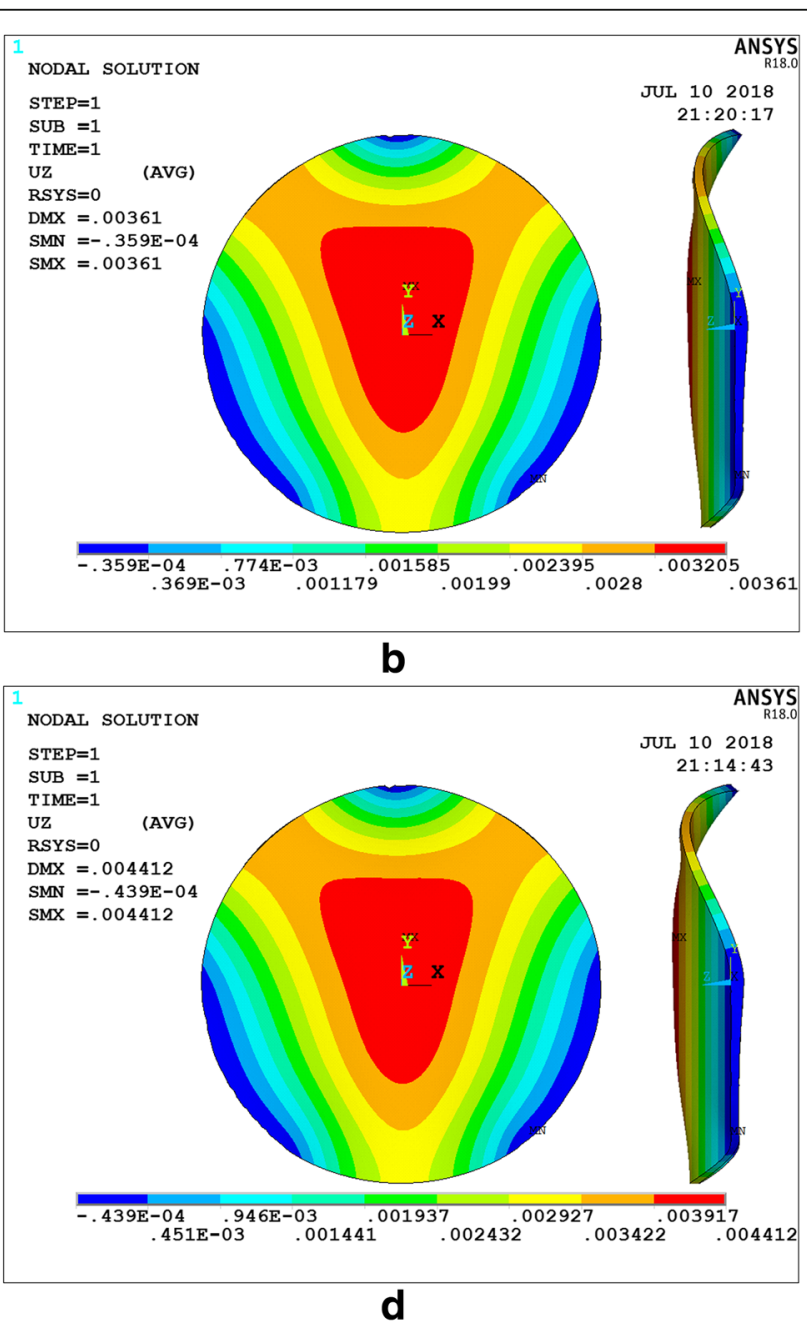

d

Fig. 4 The deformation displacement contour maps of the non-uniform PDMS membrane: a horizontal view and longitudinal view in half section on the pressure of $104 \mathrm{~Pa}$, horizontal view and longitudinal view on the pressure of (b) $90 \mathrm{~Pa}, \mathbf{c} 100 \mathrm{~Pa}$ and (d) $110 \mathrm{~Pa}$ 
the max displacement of deformation and it can be seen that the maximum deformation displacement is $3.610 \mathrm{~mm}, 4.011 \mathrm{~mm}, 4.172 \mathrm{~mm}, 4.412 \mathrm{~mm}$ under the pressure conditions of $90 \mathrm{~Pa}, 100 \mathrm{~Pa}, 104 \mathrm{~Pa}, 110 \mathrm{~Pa}$, respectively, and the corresponding volume which are needed can be calculated through the software of MATLAB using the deformation value of the membrane surface and the results are $1.6316 \mathrm{e}+3 \mathrm{~mm}^{3}, 1.8129 \mathrm{e}+$ $3 \mathrm{~mm}^{3}, 1.8854 \mathrm{e}+3 \mathrm{~mm}^{3}$ and $1.9942 \mathrm{e}+3 \mathrm{~mm}^{3}$.

From the results of simulation, the deformation rate is smaller at the top region than that at the bottom of the membrane, which indicates that the larger the applied pressure is, the higher the deflection of the non-uniform thickness membrane in the same region is. Subsequently, based on the simulation results of the PDMS membrane with the non-uniform thickness under different pressures, the power distribution of the lens can be calculated by the mean curvature calculation Eqs. 5, 6 and 7 through Matlab software, as shown in Fig. 5. It can be seen that when the pressure of liquid is exerted on the non-uniform thickness elastic membrane, the mean power distribution of the lens shows a progressively increasing trend from the top region to the bottom. When the pressure of liquid is $104 \mathrm{~Pa}$, the power of the lens increases from +4.04 to +6 progressively. Moreover, under the pressure of $90 \mathrm{~Pa}, 100 \mathrm{~Pa}$ and $110 \mathrm{~Pa}$, the power distribution characteristics are same and the power value of the lens at the distance (near) point are $+3.88(+5.77)$, $+3.48(+5.19),+4.28(+6.92)$, respectively. Also, it can be seen that the mean power distribution trends are almost the same for the same elastic membrane under the same constraint condition.

The relationship among distance/near mean power distribution, pressures on the elastic membrane and the liquid volume inside the cavity is as shown in Fig. 6. With the increasing liquid volume filling the lens' cavity formed by the PDMS membranes, which leads to an increasing curvature of the lens surface. Meanwhile, the focal lengths of the lens can be changed in different regions to realize progressive
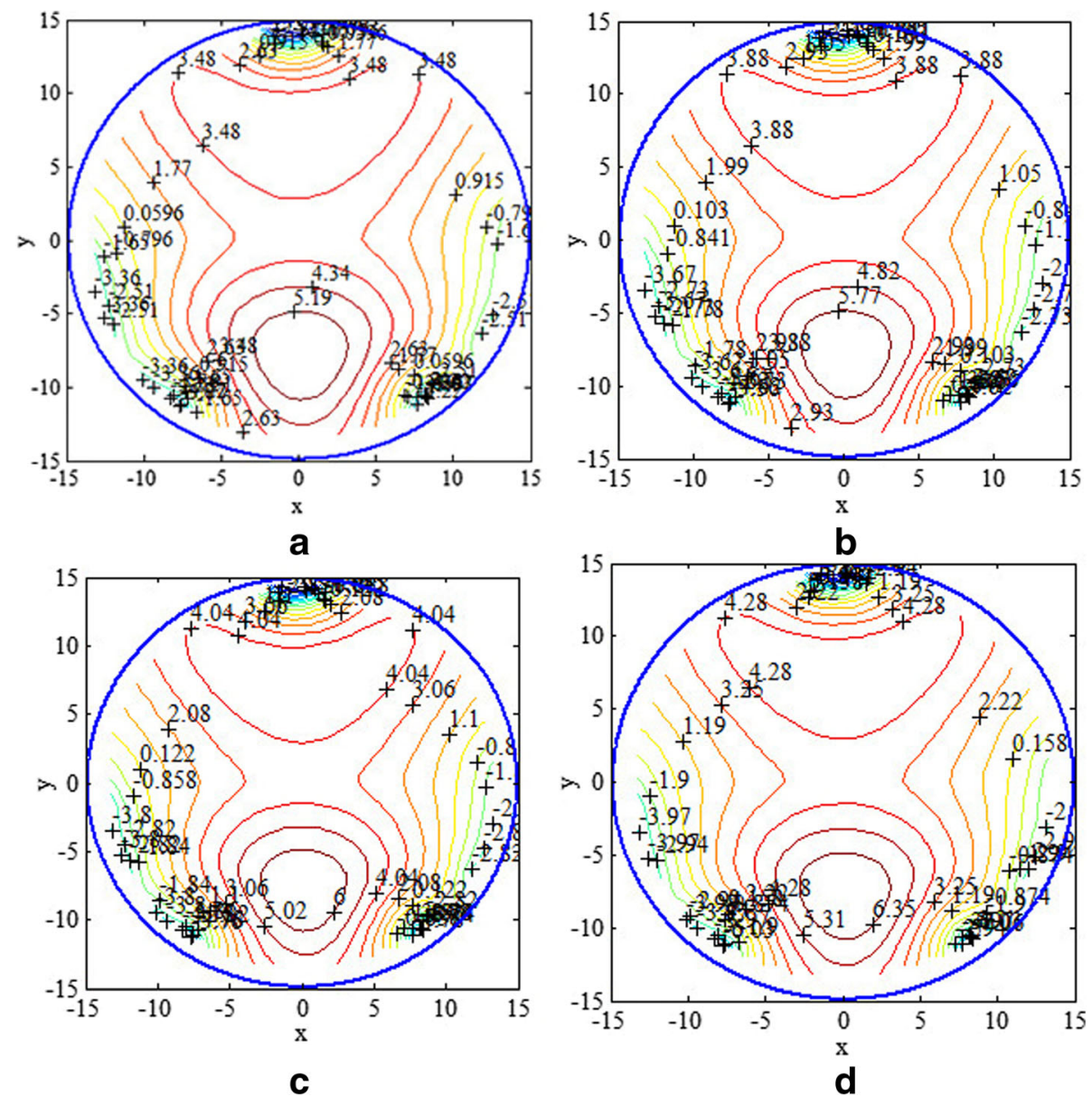

Fig. 5 The mean power distribution of the lens at different pressures: a $90 \mathrm{~Pa}$, b $100 \mathrm{~Pa}, \mathbf{c} 104 \mathrm{~Pa}$ and (d) $110 \mathrm{~Pa}$ 


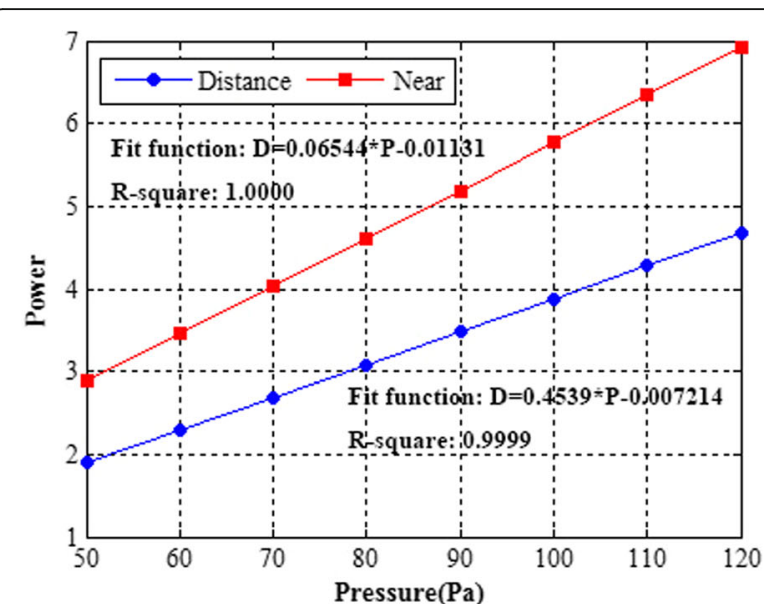

a

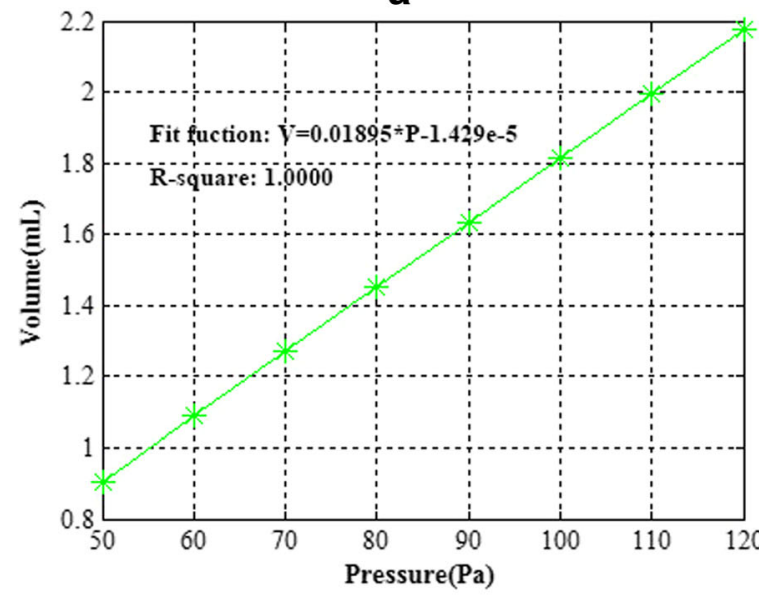

b

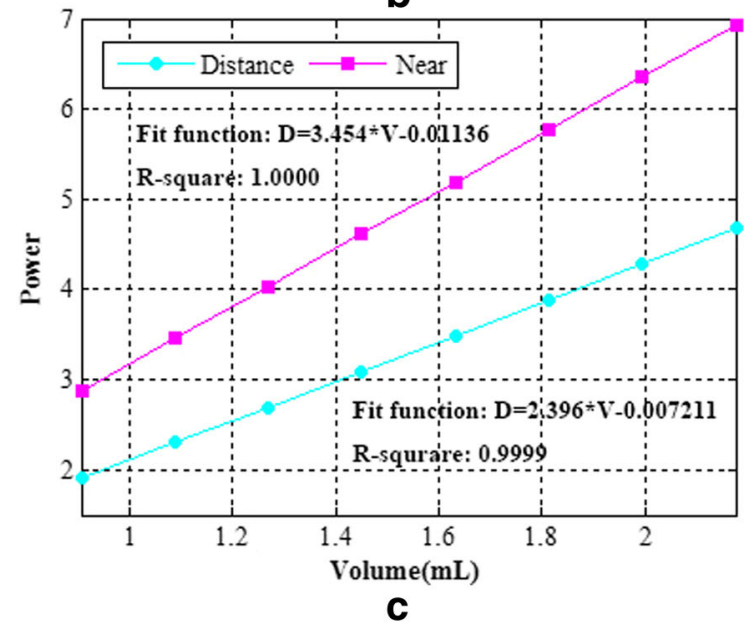

Fig. 6 The relationship among near/distance mean power distribution, pressures and volume: a pressure-power, $\mathbf{b}$ pressure-volume and (c) volume-power multifocal function. The liquid volume inlet or outlet of the cavity can be calculated by simulating the deformation of the non-uniform PDMS membrane. From the Fig. 6, it can also be concluded that the relationship among pressure, volume and power is almost linear under the pressures of $90 \mathrm{~Pa}, 100 \mathrm{~Pa}$, $104 \mathrm{~Pa}$ and $110 \mathrm{~Pa}$. Therefore, the power of the lens can be controlled by adjusting the amount of liquid inlet or outlet of the cavity of the lens linearly.

\section{Experiment}

The schematic diagram of the experimental setup and the prototype of the liquid progressive multifocal lens are shown in Fig. 7 and Fig. 8 respectively. The experimental setup for the measurement of the surface profile which mainly consists of laptop, a micro laser displacement sensor (Keyence Corporation, LK-G5001) with controller, XY-axis manual adjustment frame with two degrees freedom, the tested lens fixed by bench clamp and the optical vibration isolation platform.

A prototype of the progressive multifocal lens was made as shown in Fig. 8, the aperture of the prototype lens is $30 \mathrm{~mm}$ and the structure includes two kinds of PDMS membranes are bonded together after being treated by the oxygen plasma, the PMMA plate is as the substrate and connect with PDMS via an adhesive film, three parts of edge clamped as the constraint and the pinhole is used to input and output the liquid for the lens. In the experiment, the lens is fixed by an adjustable lens frame and placed on the $\mathrm{XY}$-axis manual adjustment frame. Furthermore, a syringe is used to pressurize the liquid into the cavity of the lens via a capillary tube and the micro laser displacement sensor is used to measure the deformation value of the lens surface.

\section{Results and discussion}

The micro laser displacement sensor is applied to measure the surface deformation of the PDMS membrane causing by the volume change of the lens, therefore the surface profile of the lens can be described through the meridian line. The chamber is filled with a varied volume of a high index optical fluid (Immersion oil $n=1.514$ ), so we know all the refractive index of the materials in the lens, the power distribution of the liquid progressive multifocal lens can be calculated from the experimental data of the membrane deformation of the lens surface, as shown in Fig. 9. It can be seen that the power of the surface at the distance point of the lens is +4.851 , and the power at the near point is +7.176 , meanwhile, the power is increased progressively along the $y$ axis, so 


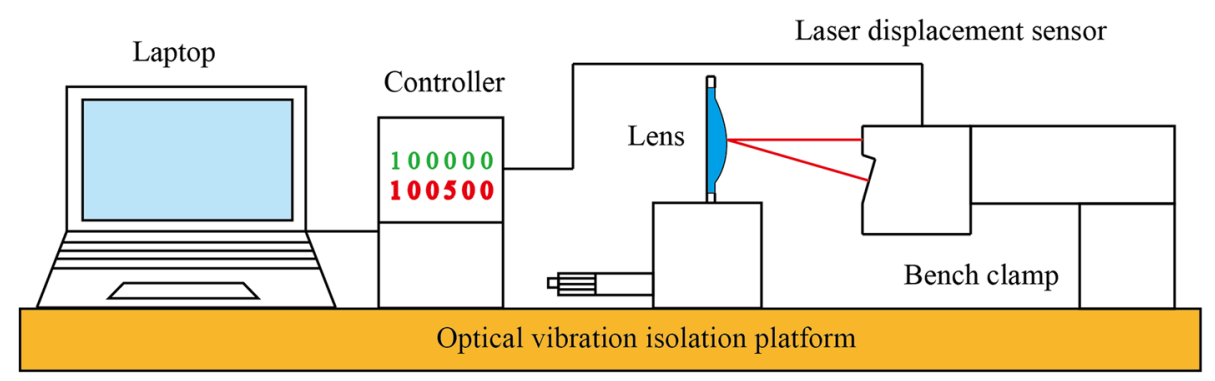

Fig. 7 Schematic diagram of experimental set-up

the trend of power distribution accords with the calculated and simulated results in Fig. 2 and Fig. 5. It can be concluded that the lens profile and the power distribution change continuously with the adjustment of the applied pressure. For the experiment, just now, we input liquid into the lens using a syringe through the pinhole and we mainly study the trend of power distribution with tiny amounts of liquid into the lens without specific volume and pressure.

\section{Conclusions}

In this paper, a novel liquid progressive multifocal lens with a non-uniform thickness elastic membrane is proposed. From the simulation and experimental investigation above, it can be concluded that the proposed liquid lens can realize progressive multifocal distribution from the top region to bottom through using non-uniform elastic membrane and the power can be adjusted by the pressure which is controlled by the liquid volume filled in the lens.

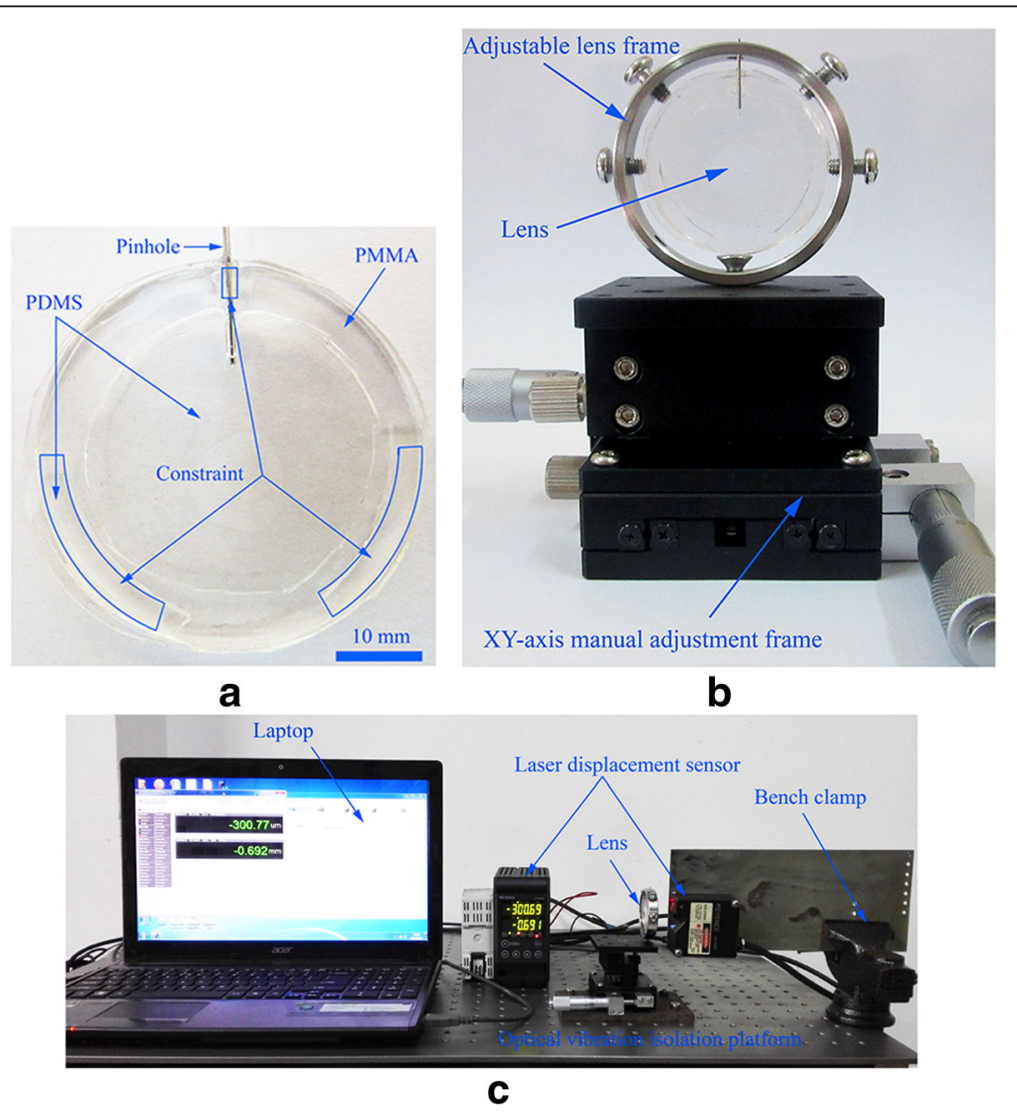

Fig. 8 A prototype and experimental set-up of the progressive multifocal lens: a liquid progressive multifocal lens, $\mathbf{b}$ regulator and (c) experimental set-up 


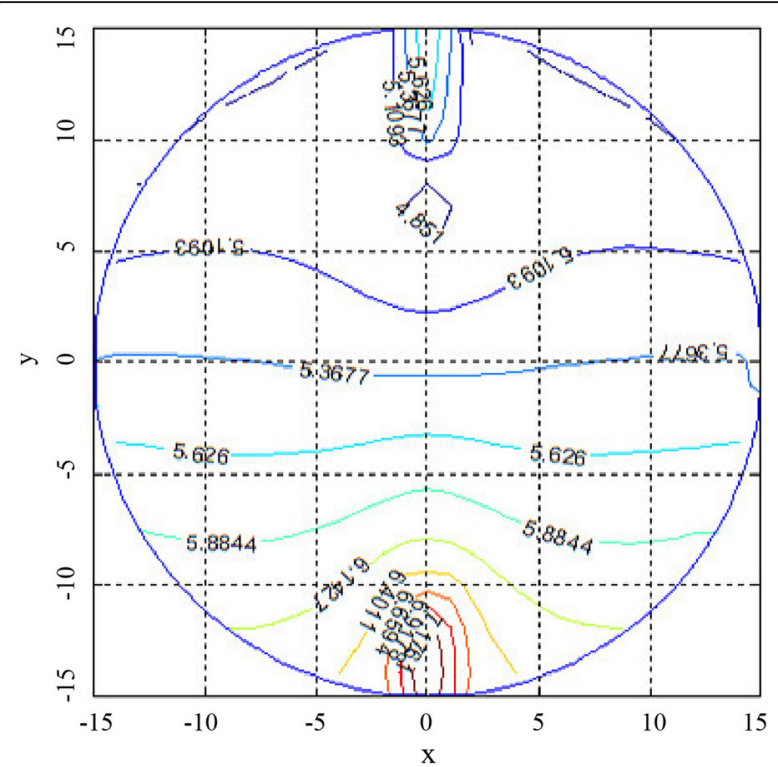

Fig. 9 The power distribution of progressive multifocal lens from the measurement

Unlike the commonly-adopted design with a constant thickness membrane as the surface of liquid lens, the membrane in the proposed design can realize that the thickness varies progressively along the meridian line. When a uniform pressure is applied on the membrane, the difference in structural parameter is then transferred to the deformed surface profile, and thereby resulting in progressive multifocal for the lens.

Although there are some deviations between the experimental and simulation results, the trend of curvature changing and power distribution still can match each other. Therefore, it would be possible to develop more applications of the lens in the other fields, such as adjustable ophthalmic eyeglasses and optical equipment in the photography. The research could be carried out in the future based on this investigation.

\section{Abbreviations}

PDMS: Polydimethylsiloxane; PMMA: Polymethyl methacrylate; FEM: Finite element method

\section{Acknowledgements}

The authors would like to give their acknowledgement to National Natural Science Foundation of China (No. 51175101) for the financial support on the paper.

\section{Availability of data and materials}

The dataset supporting the conclusions of this article is included within the article.

\section{Authors' contributions}

WJ carried out the lens structure design, simulation, experiment analysis and wrote the paper. DX helped the analysis with constructive discussions about the lens design, fabrication and provided a deeper insight into the related published work. SL built up the research project and was responsible in improving of the quality of this paper. All authors read and approved the final manuscript.

\section{Competing interests}

The authors declare that they have no competing interests.

\section{Publisher's Note}

Springer Nature remains neutral with regard to jurisdictional claims in published maps and institutional affiliations.

Received: 12 April 2018 Accepted: 16 July 2018

Published online: 31 July 2018

\section{References}

1. Kong, L.B., et al.: A theoretical and experimental investigation of design and slow tool servo machining of freeform progressive addition lenses (PALS) for optometric applications. Int. J. Adv. Manuf. Technol. 72, 33-40 (2014)

2. Savio, G., Concheri, G., Meneghello, R.: Progressive lens design by discrete shape modelling techniques. Int. J. Interact. Des. Manuf. 7, 135-146 (2013)

3. Hsu, W.-Y., et al.: Design, fabrication, and metrology of ultra-precision optical freeform surface for progressive addition lens with B-spline description. Int. J. Adv. Manuf. Technol. 63(1-4), 225-233 (2012)

4. Jiang, W., et al.: A variational-difference numerical method for designing progressive-addition lenses. Comput. Aided Des. 48, 17-27 (2014)

5. Xiang, H.Z., et al.: Theoretical and experimental investigation of design for multioptical-axis freeform progressive addition lenses. Opt. Eng. 115110(1-9), 54 (2015)

6. Shaw, D., Lin, C.W.: Design and analysis of an asymmetrical liquid-filled lens. Opt. Eng. 46, 123002 (2007)

7. Mikš, A., Novák, P.: Calculation of a surface shape of a pressure actuated membrane liquid lens. Opt. Lasers Eng. 58, 60-66 (2014)

8. $Y u, H$. , et al.: A liquid-filled tunable double-focus microlens. Opt. Express. 17, 4782-4790 (2009)

9. $\quad \mathrm{Yu}, \mathrm{H} .$, et al.: Tunable electromagnetically actuated liquid-filled lens. Sensors Actuators A Phys. 167, 602-607 (2011)

10. Sang Hoon, O., Rhee, K., Chung, S.K.: Electromagnetically driven liquid lens. Sensors Actuators A Phys. 240, 153-159 (2016)

11. Scherger, B., Jördens, C., Koch, M.: Variable-focus terahertz lens. Opt. Express. 19, 4528-4535 (2011)

12. Maffli, L., Rosset, S., Ghiardi, M., Carpi, F., Shea, H.: Ultrafast all-polymer electrically tunable silicone Lens. Adv. Funct. Mater. 25, 1656-1665 (2015)

13. Son, H.-M., Kim, M.Y., Lee, Y.-J.: Tunable-focus liquid lens system controlled by antagonistic winding-type SMA actuator. Opt. Express. 17, 14339-14350 (2009)

14. Zhao, P., Ataman, C., Zappe, H.: Spherical aberration free liquid-filled tunable lens with variable thickness membrane. Opt. Express. 23, 21264-21278 (2015)

15. Lee, J.K., Park, K.-W., Lim, G.: Variable-focus liquid Lens based on a laterallyintegrated Thermopneumatic actuator. J. Opt. Soc. Korea. 16, $22-28$ (2012)

16. Wang, L., Oku, H., Ishikawa, M.: An improved low-optical-power variable focus lens with a large aperture. Opt. Express. 22, 19448-19456 (2014)

17. PENGPENG ZHAO, ÇAGLAR ATAMAN, AND HANS ZAPPE, "Gravity-immune liquid-filled tunable lens with reduced spherical aberration," Appl. Opt. 55, 7816-7823 (2016)

18. Ren, H., Wu, S.-T.: Variable-focus liquid lens. Opt. Express. 15, 5931-5936 (2007)

19. Tan, H.Y., Loke, W.K., Nguyen, N.-T.: A reliable method for bonding polydimethylsiloxane (PDMS) to polymethylmethacrylate (PMMA) and its application in micropumps. Sensors Actuators B Chem. 151, 133-139 (2010)

20. Lee, J.K., et al.: Design and fabrication of PMMA-micromachined fluid lens based on electromagnetic actuation on PMMA-PDMS bonded membrane. J. Micromech. Microeng. 22(1-11), 115028 (2012)

21. Quanying, W., et al.: Design for progressive addition lenses. Proc. SPIE Int. Soc. Opt. Eng. 6772, 67720C (2007)

22. Quanying, W., et al.: Study on power law along meridian line for progressive addition lenses. Proc. SPIE-2008 Int. Conf. Opt. Instrum. Technol. 7156, $71561 \mathrm{~N}(2008)$

23. Winthrop, J.T.: Progressive power ophthalmic lenses. US Patent 4. 861(153), 29 (1989)

24. Yunhai, T., et al.: A kind of optimizing design method of progressive addition lenses. Proc. SPIE-5th Int. Symp. Adv. Opt. Manuf. Test. Technol. $7655,76551 \mathrm{~T}(2010)$ 